\title{
COHERENCE-BASED RECOVERY GUARANTEES FOR GENERALIZED BASIS-PURSUIT DE-QUANTIZING
}

\author{
Graeme Pope ${ }^{1}$, Christoph Studer ${ }^{2}$, and Michel Baes ${ }^{3}$ \\ ${ }^{1}$ Dept. of Inf. Technology and Electrical Engineering, ETH Zurich, Switzerland, gpope@ nari.ee.ethz.ch \\ ${ }^{2}$ Dept. of Electrical and Computer Engineering, Rice University, Houston, TX, USA, studer@ rice.edu \\ ${ }^{3}$ Institute for Operations Research, ETH Zurich, Switzerland, michel.baes@ifor.math.ethz.ch
}

\begin{abstract}
This paper deals with the recovery of signals that admit an approximately sparse representation in some known dictionary (possibly over-complete) and are corrupted by additive noise. In particular, we consider additive measurement noise with bounded $\ell_{p}$-norm for $p \geqslant 2$, and we minimize the $\ell_{q}$ quasi-norm (with $q \in(0,1]$ ) of the signal vector. We develop coherence-based recovery guarantees for which stable recovery via generalized basis-pursuit de-quantizing $\left(\mathrm{BP} \mathrm{DQ} Q_{p, q}\right)$ is possible. We finally show that depending on the measurement-noise model and the choice of the $\ell_{p}$-norm used in the constraint, $\left(\mathrm{BP} \mathrm{DQ}_{p, q}\right)$ significantly outperforms classical basis pursuit de-noising (BP DN).
\end{abstract}

Index Terms - Sparse signal recovery, sparse estimation, de-noising, de-quantizing, deterministic recovery guarantees.

\section{INTRODUCTION}

We consider the recovery of the sparse vector $\boldsymbol{x} \in \mathbb{C}^{n_{a}}$ from $m$ linear and non-adaptive measurements

$$
z=\mathbf{A} \boldsymbol{x}+\boldsymbol{n},
$$

where $\mathbf{A} \in \mathbb{C}^{m \times n_{a}}, n_{a} \geqslant m$ is a dictionary (i.e., a matrix having columns with unit $\ell_{2}$-norm) and $\boldsymbol{n} \in \mathbb{C}^{m}$ represents additive measurement noise. We consider a general noise model where $\|\boldsymbol{n}\|_{p} \leqslant \eta$ for a given $p \geqslant 2$ and some known or estimated bound $\eta$. Algorithms making use of the $\ell_{2}$-norm of the noise, i.e., with $p=2$, have been extensively studied in [1-4] for both the strictly-sparse and approximatelysparse case (see Section 2). Virtually all existing recovery guarantees rely on finding a feasible solution with smallest $\ell_{1}$-norm. However, as demonstrated in $[5,6]$, other $\ell_{q}$ quasinorms (with $q \in(0,1]$ ) can also be used-our results also give recovery guarantees for this case. The problem outlined above finds applications in, for example, image restoration [7] and de-quantization of audio or video signals [8,9].

For example, a linear quantizer $Q_{\alpha}(\cdot)$ rounds each entry of an $m$-dimensional signal vector $\boldsymbol{y}$ to the nearest integer

This work was supported in part by the Swiss National Science Foundation under Grant PA00P2-134155. The authors would like to thank C. Aubel, H. Bölcskei, R. Heckel, D. Stotz, and D. Sutter for interesting discussions. multiple of $\alpha>0$. The quantized signal is $\boldsymbol{z}=Q_{\alpha}(\boldsymbol{y})=$ $\boldsymbol{y}+\boldsymbol{n}$, where $\boldsymbol{n}$ depends on $\boldsymbol{y}$ and has maximum value $\alpha / 2$. We will show that if $\boldsymbol{y}=\mathbf{A} \boldsymbol{x}$ has a sparse representation $\boldsymbol{x}$ in dictionary $\mathbf{A}$, then we can stably recover $\boldsymbol{y}$ from $\boldsymbol{z}$ if certain conditions on $\mathbf{A}$ and the sparsity of $\boldsymbol{x}$ are satisfied.

Contributions: We develop coherence-based recovery guarantees providing conditions when we can stably recover $\boldsymbol{x}$ from $\boldsymbol{z}$ in (1) using generalized basis-pursuit de-quantizing $\left(\mathrm{BP} \mathrm{DQ} Q_{p, q}\right)$. In particular, we extend existing coherencebased recovery guarantees to the more general case $p \geqslant 2$ and $0<q \leqslant 1$. Finally, we provide simulation results to demonstrate the importance of choosing the $\ell_{p}$-norm in accordance with the considered measurement-noise model.

Notation: The number of nonzero components of the vector $\boldsymbol{x}$ (the sparsity) is denoted by $n_{x}=\|\boldsymbol{x}\|_{0}$ and $\operatorname{supp}_{k}(\boldsymbol{x})$ designates the indices of the $k$ largest components (in magnitude) of $\boldsymbol{x}$; when $\mathcal{S}$ is a set of indices, $x_{\mathcal{S}}$ is the vector that agrees with $\boldsymbol{x}$ on $\mathcal{S}$ and is 0 elsewhere. The coherence $\mu$ of A is defined as $\mu \triangleq \max _{i \neq j}\left|\left\langle\boldsymbol{a}_{i}, \boldsymbol{a}_{j}\right\rangle\right|$, where $\boldsymbol{a}_{i}$ is the $i$ th column of $\mathbf{A}$.

\section{BACKGROUND}

As mentioned above, the special case $p=2$ was investigated previously in [1-4]. The following well-known result guarantees the stable recovery of approximately sparse signals $\boldsymbol{x}$ from $\mathbf{z}$ in (1) using basis pursuit de-noising (BP DN).

Theorem 1 ([3, Thm. 2.1] and [4, Thm. 1]). Let $\boldsymbol{z}=\mathbf{A} \boldsymbol{x}+\boldsymbol{n}$ with $\|\boldsymbol{n}\|_{2} \leqslant \eta$ and $\mathcal{X}=\operatorname{supp}_{n_{x}}(\boldsymbol{x})$. If

$$
n_{x}=\|\boldsymbol{x}\|_{0}<\left(1+\mu^{-1}\right) / 2,
$$

then the solution $\hat{\boldsymbol{x}}$ of

$$
(\mathrm{BP} D \mathrm{DN}) \quad \operatorname{minimize}\|\hat{\boldsymbol{x}}\|_{1} \text { s.t. }\|\boldsymbol{z}-\mathbf{A} \hat{\boldsymbol{x}}\|_{2} \leqslant \varepsilon_{2},
$$

with $\eta \leqslant \varepsilon_{2}$ satisfies

$$
\|\boldsymbol{x}-\hat{\boldsymbol{x}}\|_{2} \leqslant \mathrm{C}_{0}\left(\varepsilon_{2}+\eta\right)+\mathrm{C}_{1}\left\|\boldsymbol{x}-\boldsymbol{x}_{\mathcal{X}}\right\|_{1},
$$

where $\mathrm{C}_{0}$ and $\mathrm{C}_{1}$ are constants that depend only on $\mu$ and $n_{x}$.

In the absence of measurement noise, we have $\eta=0$, which, for $\varepsilon_{2}=0$, gives the classical (BP) recovery result [1]. 
Related recovery guarantees were also developed in [10] and are based on the restricted isometry constant (RIC) $\delta_{k}$ of the dictionary A. For example, in [9], the (BP DN) problem was extended to basis-pursuit de-quantizing

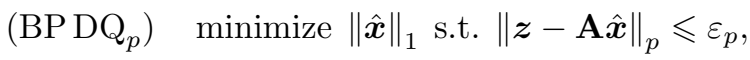

for $p \geqslant 2$. Corresponding recovery guarantees require the extended restricted isometry property (RIP) $(p, q)$ of order $k$. A matrix $\mathbf{A}$ is said to satisfy the extended RIP if there exist constants $\delta_{k}>0$ and $\mu_{p, q}>0$ such that [9]

$$
\mu_{p, q}^{q}\left(1-\delta_{k}\right)\|\boldsymbol{x}\|_{q}^{q} \leqslant\|\mathbf{A} \boldsymbol{x}\|_{p}^{q} \leqslant \mu_{p, q}^{q}\left(1+\delta_{k}\right)\|\boldsymbol{x}\|_{q}^{q},
$$

for every $\|\boldsymbol{x}\|_{0} \leqslant k$. The following theorem guarantees stable recovery using $\left(\mathrm{BP} \mathrm{DQ}_{p}\right)$.

Theorem 2 ([9, Thm. 2]). Let $\boldsymbol{z}=\mathbf{A} \boldsymbol{x}+\boldsymbol{n}$ with $\|\boldsymbol{n}\|_{p} \leqslant \varepsilon_{p}$ and $\mathcal{X}=\operatorname{supp}_{n_{x}}(\boldsymbol{x})$. If the extended RIP with $(p, 2)$ of order $3 n_{x}$ is satisfied, then the solution $\hat{\boldsymbol{x}}$ of $\left(\mathrm{BP} \mathrm{DQ}_{p}\right)$ satisfies

$$
\|\boldsymbol{x}-\hat{\boldsymbol{x}}\|_{2} \leqslant \mathrm{C}_{2} \varepsilon_{p}+\mathrm{C}_{3}\left\|\boldsymbol{x}-\boldsymbol{x}_{\mathcal{X}}\right\|_{1},
$$

where $\mathrm{C}_{2}$ and $\mathrm{C}_{3}$ depend only on $\delta_{n_{x}}, \delta_{2 n_{x}}$ and $\delta_{3 n_{x}}$.

The major drawback of RIP-based recovery guarantees is the fact that no deterministic construction is known that lead to matrices satisfying the RIP. One attempt to arrive at recovery guarantees that can be computed efficiently are obtained by bounding the extended RIP in terms of the coherence parameter $\mu$. For $p \geqslant 2, q=1$, and $k=3 n_{x}$, it follows from Lemmas 4 and 6 that $\delta_{3 n_{x}} \leqslant \mu\left(3 n_{x}-1\right)$ in (3), implying that the matrix has the extended $\operatorname{RIP}(p, 2)$ of order $3 n_{x}$ if

$$
n_{x}<\left(1+\mu^{-1}\right) / 3 \text {. }
$$

We emphasize, however, that this recovery condition is stricter than that for (BP DN) in (2). We next show that a direct proof for $\left(\mathrm{BP} \mathrm{DQ} Q_{p}\right)$ improves upon (4) and results in the same coherence-based recovery condition as for (BP DN).

\section{COHERENCE-BASED RECOVERY GUARANTEES}

We consider a generalized version of $\left(\mathrm{BP} \mathrm{DQ}_{p}\right)$, referred to as generalized basis pursuit de-quantizing $\left(\mathrm{BP} \mathrm{DQ}_{p, q}\right)$, where we minimize the $\ell_{q}$ quasi-norm (for $0<q \leqslant 1$ ) of the vector $\hat{\boldsymbol{x}}$

$$
\left(\mathrm{BP} \mathrm{DQ}_{p, q}\right) \quad \text { minimize }\|\hat{\boldsymbol{x}}\|_{q} \text { s.t. }\|\boldsymbol{z}-\mathbf{A} \hat{\boldsymbol{x}}\|_{p} \leqslant \varepsilon_{p} .
$$

The following theorem guarantees the stable recovery of $\boldsymbol{x}$ using $\left(\mathrm{BP} \mathrm{DQ}_{p, q}\right)$ if (5) is satisfied.

Theorem 3. Let $\boldsymbol{z}=\mathbf{A} \boldsymbol{x}+\boldsymbol{n}$ with $\|\boldsymbol{n}\|_{p} \leqslant \eta$ and $\mathcal{X}=$ $\operatorname{supp}_{n_{x}}(\boldsymbol{x})$. If

$$
n_{x}<\frac{1}{1+\alpha_{q} \beta_{q}}\left(1+\mu^{-1}\right),
$$

where $\alpha_{q}=2^{1 / q-1}$ and $\beta_{q}=\left(n_{x}\right)^{1 / q-1}$, then the solution, $\hat{\boldsymbol{x}}$, of $\left(\mathrm{BP} \mathrm{DQ}_{p, q}\right)$ with $\eta \leqslant \varepsilon_{p}$ satisfies

$$
\|\boldsymbol{x}-\hat{\boldsymbol{x}}\|_{2} \leqslant \mathrm{C}_{4} m^{\frac{1}{2}-\frac{1}{p}}\left(\eta+\varepsilon_{p}\right)+\mathrm{C}_{5}\left\|\boldsymbol{x}-\boldsymbol{x}_{\mathcal{X}}\right\|_{1},
$$

where $\mathrm{C}_{4}$ and $\mathrm{C}_{5}$ depend only on $\mu, n_{x}$ and $q$, but not on $p$.

Proof. See Appendix A for the proof.

Note that in the case $q=1$, we have $\alpha_{q} \beta_{q}=1$, and (5) gives the same condition as (2). Moreover, for $q=1$ and $p=2$, we get the same constants as in Theorem 1. Hence, Theorem 3 results in a better (i.e., less restrictive) recovery guarantee than that obtained in (4).

An important question to ask is, how does this result compare to the existing results for (BPDN) given that they both have the same condition for stable recovery? Do we get an improvement by using a different norm constraint? To this end, we consider the quantization example given in Section 1, with $\alpha=2 \tau$. In this case, $\|\boldsymbol{n}\|_{\infty} \leqslant \tau$ and $\|\boldsymbol{n}\|_{2} \leqslant \sqrt{m} \tau$, and by setting $\varepsilon_{\infty}=\tau$, we get the recovery condition

$$
\left\|\boldsymbol{x}-\hat{\boldsymbol{x}}_{\infty}\right\|_{2} \leqslant 2 \mathrm{C}_{0} \sqrt{m} \tau+\mathrm{C}_{1}\left\|\boldsymbol{x}-\boldsymbol{x}_{\mathcal{X}}\right\|_{1} .
$$

But applying Theorem 1 with $\varepsilon_{2}=\sqrt{m} \tau$ leads to same recovery bound as in (7), implying that using a more suitable norm does not necessarily result in better analytical recovery guarantees. However, as shown next, the use of $\left(B P D Q_{p}\right)$ can give vastly superior recovery performance in practice.

\section{SIMULATION RESULTS}

\subsection{Comparison of different norms}

We first demonstrate the impact of the $\ell_{p}$-norm on the recovery performance. Take $\mathbf{A}$ to be a randomly sub-sampled Hadamard matrix of dimension $128 \times 256$, and assume $\boldsymbol{x}$ has $n_{x}=8$ non-zero entries. The measurement noise is generated according to the following models: (i) i.i.d. Gaussian with zero mean, (ii) uniformly distributed random noise on an interval or discrete set, and (iii) noise as obtained via quantization. In each case, we normalize the signal to have unit energy and choose the noise variance so that the expected signal-to-noise-ratio SNR is $10 \mathrm{~dB}$. Note that we only consider the case $q=1$, since for $q<1$, $\left(\mathrm{BPDQ}_{p, q}\right)$ is not a convex optimization problem. We use CVX [11] to solve the optimization problems and consider a genie-aided norm bound $\varepsilon_{p}=\|\boldsymbol{n}\|_{p}$, which is the smallest bound guaranteeing that the correct solution lies in the feasible set.

In Fig. 1, we plot the mean-square error MSE, obtained by averaging $\|\boldsymbol{x}-\hat{\boldsymbol{x}}\|_{2}^{2} /\|\boldsymbol{x}\|_{2}^{2}$ over 1000 Monte-Carlo trials. For each noise model, we plot the MSE relative to the norm constraint that gives the lowest MSE for that noise model. Observe that as the noise becomes less concentrated around the mean, higher order norms seem to perform better e.g., when the noise is uniformly distributed on $[-1,1]$, the $\ell_{4}$ norm constraint performs best, but as the noise clusters around \pm 1 , e.g., 


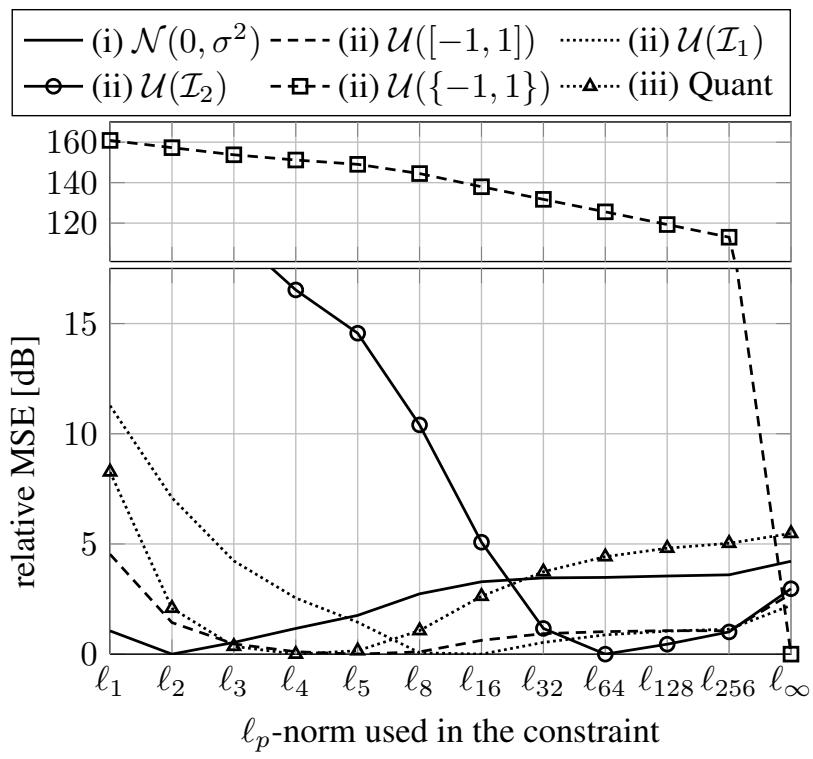

Fig. 1. Relative MSE for different noise models. "Quant" refers to quantization noise, $\mathcal{I}_{1}=[-1,-0.5] \cup[0.5,1]$ and $\mathcal{I}_{2}=[-1,-0.9] \cup[0.9,1]$. Here, $n_{x}=8$ is considered; similar behavior is observed for other sparsity levels.

with $\mathcal{U}\left(\mathcal{I}_{2}\right)$, the $\ell_{64}$ norm gives better performance. This implies that the choice of the norm to be used in the constraint of $\left(\mathrm{BP} \mathrm{DQ}_{p, q}\right)$ is important and should be chosen in accordance with the considered measurement noise model.

\subsection{De-quantization of band-limited audio signals}

We now reconstruct a quantized audio signal. We take $m=$ 256 sample segments of Mozart's Quintet K.452 at random, quantize it to 4 bit precision and reconstruct using $\left(\mathrm{BP} \mathrm{DQ}_{p}\right)$. We plot the resulting SNR $=\|\boldsymbol{y}\|_{2} /\|\mathbf{A} \hat{\boldsymbol{x}}-\boldsymbol{y}\|_{2}$ ( $\boldsymbol{y}$ is the unquantized signal) in Fig. 2, relative to the performance of (BP DN). We use two discrete cosine transform (DCT) matrices of dimension $m \times n(n=256$ or 512$)$ as dictionaries. When $n>m$, the dictionary is overcomplete and may lead to a sparser vector $\boldsymbol{x}$. Note that here we deal with approximately sparse vectors. If we know $\|\boldsymbol{n}\|_{p}$ (which is often not the case), then using the $\ell_{3}$ norm offers slightly improved reconstruction SNR $(\approx 0.5 \mathrm{~dB})$. However, if we only have an upper-bound for the noise, e.g., $\varepsilon_{p}=2^{-4} \mathrm{~m}^{1 / p}$ (which is the $\ell_{p}$-norm of the noise in the worse case scenario), then we see that higherorder norms offer a significant improvement over (BP DN). Using an overcomplete DCT matrix, seems to make little difference, when compared to the standard DCT matrix.

\section{CONCLUSION}

In this paper, we have generalized the coherence-based results of $[3,4]$ to include the cases where a sparse signal is corrupted by noise with bounded $\ell_{p}$ norm $(p \geqslant 2)$. We have shown that by using the appropriate optimization program, we

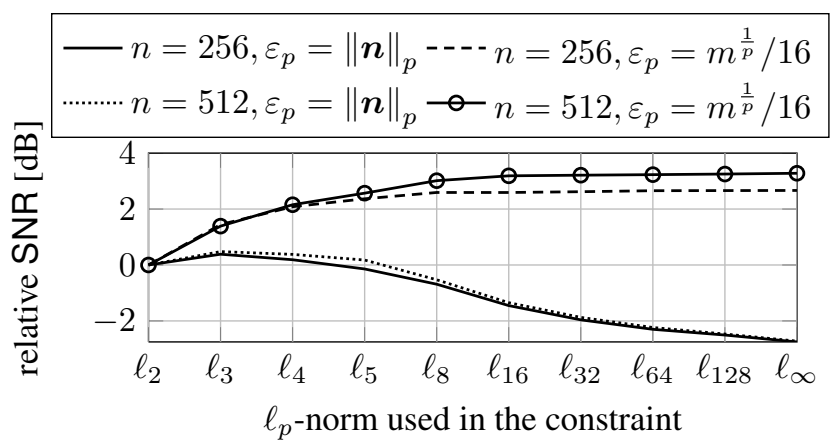

Fig. 2. Reconstruction SNR (relative to the $\ell_{2}$ case) of an audio signal recovered after 4 bit quantization.

can achieve improved recovery performance when compared to solving (BP DN). Furthermore, our analytical guarantees also enable the stable recovery if we minimize the $\ell_{q}$ quasinorm $(0<q \leqslant 1)$ of $\boldsymbol{x}$. We presented simulation results to demonstrate that the choice of the norm employed in the constraint is an important design consideration and should be chosen in accordance with the measurement noise model for optimum performance.

\section{A. PROOF OF THEOREM 3}

We now present the proofs of our results for $\left(\mathrm{BP} \mathrm{DQ}_{p, q}\right)$. In this section, the $d u a l p^{\prime}$ of a number $p \in[1, \infty]$ satisfies $1 / p+$ $1 / p^{\prime}=1$. We first restate a number of well-known bounds.

Lemma 4 (Section 2.8 of [12]). For any $\boldsymbol{x} \in \mathbb{C}^{m}, 1 \leqslant p \leqslant$ $s \leqslant \infty$ with duals $p^{\prime}$ and $s^{\prime}$

$$
\|\boldsymbol{x}\|_{p} m^{1 / p^{\prime}} \leqslant\|\boldsymbol{x}\|_{s} m^{1 / s^{\prime}} .
$$

Lemma 5. For any $\boldsymbol{a}, \boldsymbol{b} \in \mathbb{C}^{m}, 0<q \leqslant 1, p \geqslant 1$ and $\alpha_{q} \triangleq 2^{1 / q-1}$, we have

$$
\begin{aligned}
\|\boldsymbol{a}\|_{1} & \leqslant\|\boldsymbol{a}\|_{q} \leqslant m^{\frac{1}{q}-\frac{1}{p}}\|\boldsymbol{a}\|_{p} \\
\left(|a|^{q}+|b|^{q}\right)^{\frac{1}{q}} & \leqslant \alpha_{q}|a|+\alpha_{q}|b| \\
\|\boldsymbol{a}\|_{q}^{q}-\|\boldsymbol{b}\|_{q}^{q} & \leqslant\|\boldsymbol{a}-\boldsymbol{b}\|_{q}^{q} \\
\|\boldsymbol{a}+\boldsymbol{b}\|_{q} & \leqslant \alpha_{q}\left(\|\boldsymbol{a}\|_{q}+\|\boldsymbol{b}\|_{q}\right) .
\end{aligned}
$$

Proof. Equation (9a) is in [12, §2.8] and (9b) follows from the case with $m=2$ and $p=1$. Equation (9c) follows by applying Jensen's inequality to the mapping $t \mapsto t^{q}$. Finally, (9d) is a consequence of (9b) and (9c).

Lemma 6. Let $\mathbf{A} \in \mathbb{C}^{m \times n_{a}}$ have columns of unit $\ell_{2}$ norm. Let $\mu$ be its coherence and $\boldsymbol{x} \in \mathbb{C}^{n_{a}} \backslash\{\mathbf{0}\}$ be $n_{x}$-sparse. Then

$$
\|\boldsymbol{x}\|_{2}^{2}\left(1-\mu\left(n_{x}-1\right)\right) \leqslant\|\mathbf{A} \boldsymbol{x}\|_{2}^{2} \leqslant\|\boldsymbol{x}\|_{2}^{2}\left(1+\mu\left(n_{x}-1\right)\right) .
$$

Proof. The proof follows by bounding the off-diagonal entries of $\mathbf{A}^{*} \mathbf{A}$ with the coherence parameter $\mu$ and applying Geršgorin's disk theorem [13, Thm. 6.1.1]. 
Proof of Theorem 3. Set $\boldsymbol{h} \triangleq \hat{\boldsymbol{x}}-\boldsymbol{x}$, and let $\mathcal{X}$ be the support of the best $n_{x}$-sparse approximation to the signal $\boldsymbol{x}$. The goal is to bound $\|\boldsymbol{h}\|_{2}$. Write $\mathbf{A}^{*} \mathbf{A}=\mathbf{1}+\mathbf{M}$, so that $\mathbf{M}$ has a zero diagonal, then

$$
\|\mathbf{A} \boldsymbol{h}\|_{2}^{2}=\|\boldsymbol{h}\|_{2}^{2}+\boldsymbol{h}^{*} \mathbf{M} \boldsymbol{h} \geqslant\|\boldsymbol{h}\|_{2}^{2}-\mu\left(\|\boldsymbol{h}\|_{1}^{2}-\|\boldsymbol{h}\|_{2}^{2}\right) .
$$

Rearranging this result leads to

$$
\|\boldsymbol{h}\|_{2}^{2} \leqslant \frac{\|\mathbf{A} \boldsymbol{h}\|_{2}^{2}+\mu\|\boldsymbol{h}\|_{1}^{2}}{1+\mu} \stackrel{(8)}{\leqslant} \frac{\gamma_{p}^{2}\|\mathbf{A} \boldsymbol{h}\|_{p}^{2}+\mu\|\boldsymbol{h}\|_{1}^{2}}{1+\mu}
$$

with $\gamma_{p}=m^{\frac{1}{2}-\frac{1}{p}}$. First we have the so-called tube constraint

$$
\|\mathbf{A} \boldsymbol{h}\|_{p} \leqslant\|\mathbf{A} \boldsymbol{x}-\boldsymbol{z}\|_{p}+\|\mathbf{A} \hat{\boldsymbol{x}}-\boldsymbol{z}\|_{p} \leqslant \eta+\varepsilon_{p}
$$

Since $\hat{\boldsymbol{x}}$ is a minimizer, we have

$$
\begin{aligned}
\|\boldsymbol{x}\|_{q}^{q} & =\left\|\boldsymbol{x}_{\mathcal{X}^{c}}\right\|_{q}^{q}+\left\|\boldsymbol{x}_{\mathcal{X}}\right\|_{q}^{q} \geqslant\|\hat{\boldsymbol{x}}\|_{q}^{q} \\
& =\left\|\boldsymbol{h}_{\mathcal{X}^{c}}+\boldsymbol{x}_{\mathcal{X}^{c}}\right\|_{q}^{q}+\left\|\boldsymbol{h}_{\mathcal{X}}+\boldsymbol{x}_{\mathcal{X}}\right\|_{q}^{q} \\
& \stackrel{(9 \mathrm{c})}{\geqslant}\left\|\boldsymbol{h}_{\mathcal{X}^{c}}\right\|_{q}^{q}-\left\|\boldsymbol{x}_{\mathcal{X}^{c}}\right\|_{q}^{q}-\left\|\boldsymbol{h}_{\mathcal{X}}\right\|_{q}^{q}+\left\|\boldsymbol{x}_{\mathcal{X}}\right\|_{q}^{q},
\end{aligned}
$$

which corresponds to the cone constraint and

$$
2\left\|\boldsymbol{x}_{\mathcal{X}^{c}}\right\|_{q}^{q}+2\left\|\boldsymbol{h}_{\mathcal{X}}\right\|_{q}^{q} \geqslant\left\|\boldsymbol{h}_{\mathcal{X}^{c}}\right\|_{q}^{q}+\left\|\boldsymbol{h}_{\mathcal{X}}\right\|_{q}^{q}=\|\boldsymbol{h}\|_{q}^{q} .
$$

With (9b), we arrive at

$$
2^{\frac{2}{q}-1}\left(\left\|\boldsymbol{x}_{\mathcal{X}^{c}}\right\|_{q}+\left\|\boldsymbol{h}_{\mathcal{X}}\right\|_{q}\right) \geqslant\|\boldsymbol{h}\|_{q} .
$$

To bound $\left\|\boldsymbol{h}_{\mathcal{X}}\right\|_{q}$ set $\kappa_{x}=\sqrt{1+\mu\left(n_{x}-1\right)}$ and $\theta_{x}=$ $n_{x}^{1 / q-1 / 2} /\left(1-\mu\left(n_{x}-1\right)\right)$, which enables us to write

$$
\begin{aligned}
& \left\|\boldsymbol{h}_{\mathcal{X}}\right\|_{2}\left\|\boldsymbol{h}_{\mathcal{X}}\right\|_{q} \stackrel{(9 \mathrm{a})}{\leqslant} n_{x}^{\frac{1}{q}-\frac{1}{2}}\left\|\boldsymbol{h}_{\mathcal{X}}\right\|_{2}^{2} \leqslant \theta_{x}\left\|\mathbf{A} \boldsymbol{h}_{\mathcal{X}}\right\|_{2}^{2} \\
& =\theta_{x}\left(\mathbf{A} \boldsymbol{h}^{*}\left(\mathbf{A} \boldsymbol{h}_{\mathcal{X}}\right)-\theta_{x}\left(\mathbf{A} \boldsymbol{h}_{\mathcal{X}^{c}}\right)^{*}\left(\mathbf{A} \boldsymbol{h}_{\mathcal{X}}\right)\right. \\
& \leqslant \theta_{x}\|\mathbf{A} \boldsymbol{h}\|_{2}\left\|\mathbf{A} \boldsymbol{h}_{\mathcal{X}}\right\|_{2}+\theta_{x} \mu\left\|\boldsymbol{h}_{\mathcal{X}^{c}}\right\|_{1}\left\|\boldsymbol{h}_{\mathcal{X}}\right\|_{1} \\
& \stackrel{(9 \mathrm{a})}{\leqslant} \theta_{x}\left\|\boldsymbol{h}_{\mathcal{X}}\right\|_{2}\left(\kappa_{x} \gamma_{p}\left(\eta+\varepsilon_{p}\right)+\mu \sqrt{n_{x}}\left\|\boldsymbol{h}_{\mathcal{X}^{c}}\right\|_{q}\right) .
\end{aligned}
$$

Now, we can use (12) and (9b) to bound $\left\|\boldsymbol{h}_{\mathcal{X}^{c}}\right\|_{q}$

$$
\left\|\boldsymbol{h}_{\mathcal{X}^{c}}\right\|_{q} \leqslant 2^{\frac{1}{q}-1}\left(2^{\frac{1}{q}}\left\|\boldsymbol{x}_{\mathcal{X}^{c}}\right\|_{q}+\left\|\boldsymbol{h}_{\mathcal{X}}\right\|_{q}\right) .
$$

Combining with (14) and (9a) we arrive at

$$
\left(1-\theta_{x} \mu \alpha_{q} n_{x}^{\frac{1}{2}}\right)\left\|\boldsymbol{h}_{\mathcal{X}}\right\|_{q} \leqslant \theta_{x}\left(\kappa_{x} \gamma_{p}\left(\eta+\varepsilon_{p}\right)+\mu \alpha_{q} n_{x}^{\frac{1}{2}}\left\|\boldsymbol{x}_{\mathcal{X}^{c}}\right\|_{q}\right) .
$$

If we assume that $1-\theta_{x} \mu 2^{\frac{1}{q}-1}>0$, that is

$$
\frac{1}{\mu}+1>\left(n_{x}\right)^{\frac{1}{q}} 2^{\frac{1}{q}-1}+n_{x}=n_{x}\left(\left(2 n_{x}\right)^{\frac{1}{q}-1}+1\right)
$$

which implies (5). We obtain the desired result by combining the last inequality with (10), (11), and (13), and with constants

$$
\begin{aligned}
\mathrm{C}_{0} & =\frac{1}{\sqrt{1+\mu}}+\frac{2^{\frac{1}{q}} \alpha_{q} \sqrt{\mu n_{x}} \sqrt{1+\mu\left(n_{x}-1\right)}}{\sqrt{1+\mu}\left(1-\mu\left(\left(1+\alpha_{q} \beta_{q}\right) n_{x}-1\right)\right)} \\
\mathrm{C}_{1} & =\frac{\alpha_{q \sqrt{\mu}}\left[\mu n_{x}\left(\alpha_{q} 2^{\frac{1}{q}}-1-\alpha_{q} \beta_{q}\right)+1+\mu\right]}{\sqrt{1+\mu}\left(1-\mu\left(\left(1+\beta_{q} \alpha_{q}\right) n_{x}-1\right)\right)},
\end{aligned}
$$

where $\alpha_{q}=2^{1 / q-1}$ and $\beta_{q}=n_{x}^{1 / q-1}$.

\section{B. REFERENCES}

[1] David Donoho, Michael Elad, and V N Temlyakov, "Stable recovery of sparse overcomplete representations in the presence of noise," IEEE Trans. Inf. Theory, vol. 52 , no. 1 , pp. $6-18,2006$.

[2] J.-J Fuchs, "Recovery of exact sparse representations in the presence of bounded noise," IEEE Trans. Inf. Theory, vol. 51, no. 10, pp. 3601 - 3608, 2005.

[3] Tony Cai, Lie Wang, and Guangwu Xu, "Stable recovery of sparse signals and an oracle inequality," IEEE Trans. Inf. Theory, vol. 56, no. 7, pp. 3516-3522, 2010.

[4] Christoph Studer and Richard G Baraniuk, "Stable restoration and separation fo approximately sparse signals," Submitted Appl. Comput. Harmon. Anal., 2011.

[5] David Donoho, “Compressed sensing," IEEE Trans. Inf. Theory, vol. 52, no. 4, pp. 1289-1306, 2006.

[6] Simon Foucart and Ming-Jun Lai, "Sparsest solutions of underdetermined linear systems via $\ell_{q}$-minimization for $0<q \leqslant 1$," Appl. Comput. Harmon. Anal., Jan 2009.

[7] $\mathrm{P}$ Weiss, L Blanc-Feraud, $\mathrm{T}$ Andre, and $\mathrm{M}$ Antonini, "Compression artifacts reduction using variational methods : Algorithms and experimental study," ICASSP 2008, pp. 1173 - 1176, 2008.

[8] Argyrios Zymnis, Stephen P Boyd, and Emmanuel J Candès, "Compressed sensing with quantized measurements," Signal Processing Letters, IEEE, vol. 17, no. 2, pp. $149-152,2010$.

[9] Laurent Jacques, David K Hammond, and M Jalal Fadili, "Dequantizing compressed sensing: When oversampling and non-gaussian constraints combine," IEEE Trans. Inf. Theory, vol. 57, no. 1, pp. 559- 571, 2011.

[10] Emmanuel J Candès, Justin Romberg, and Terrence Tao, "Stable signal recovery from incomplete and inaccurate measurements," Comm. Pure Appl. Math., vol. 59, no. 8, pp. 1207-1223, 2006.

[11] M Grant and Stephen P Boyd, "CVX: Matlab software for disciplined convex programming (web page and software)," 2009.

[12] Godfrey H Hardy, John E Littlewood, and George Pólya, Inequalities, Cambridge Univ. Press, Jan 1997.

[13] R Horn and C Johnson, Matrix analysis, Cambridge Univ. Press, Jan 1990. 